\title{
The Development of Mobile Short Video Communication in the Context of the Mobile Internet
}

\author{
Huixia Wang, ${ }^{1, *}$ Sa Yang, ${ }^{2}$ Zhaoxia Dai ${ }^{3}$ \\ 1. School of Educational Sciences, Jiangsu Second Normal University, Nanjing 210013, Jiangsu, China \\ 2. National Engineering Research Center for E-Learning, Central China Normal University, Wuhan 430079, Hubei, China \\ 3. Huanggang Normal University, Huanggang 438000, Hubei, China \\ *: All correspondence should be sent to: Ms. Huixia Wang. \\ Author's Contact: Huixia Wang, E-mail: hellon 123@163.com; Sa Yang, E-mail: 794228513@qq.com; Zhaoxia Dai, E-mail: \\ 158015775@qq.com \\ Funding: The innovative research of animation creation inheriting National Culture (Project \#: 2020SJZDA029), university philosophy and social \\ science research major project in Jiangsu; and Research on classroom teaching evaluation based on the model of one school with multi-point \\ delivery (Project \#: 19Y120), Humanities and Social Science Project of Hubei Education Department. \\ DOI: https://doi.org/10.15354/si.22.re011 \\ The authors declare no competing interest
}

In the context of the mobile Internet, continuously upgraded media and increasing numbers of terminal devices has enabled information communication to become instant and abundant. The lifestyle of the public has also changed with the evolution of the Internet. The public's growing enthusiasm for watching short videos on smart phones are one example. This paper discusses changes in the communication environment, namely the concept and development of mobile short videos. Application of Lasswell's " 5 W" model to mobile short video communication, is used to analyze this form of communication, using Meipai, a Chinese mobile short video application, as a case study. Suggestions for potential development in this area are also considered.

Keywords: Mobile Short Video; Communication; 5W Communication Model; Disseminator; Audience Science Insights, 2022 January 17; Vol. 40, No. 1, pp.421-426.

(c) 2022 Insights Publisher. All rights reserved.

Creative Commons Non Commercial CC BY-NC: This article is distributed under the terms of the Creative Commons Attribution-NonCommercial 4.0 License which permits non-commercial use, reproduction and distribution of the work without further permission provided the original work is attributed by the Insights Publisher.

$\mathrm{W}$ ITH the continued innovation of Internet technology, new media vehicles such as smart phones, augmented reality, virtual reality and tablet computers are now widely used by many people in daily life. This "mobile Internet" is beneficial for individuals as it allows interaction, experience and sharing, and for many people has made the Internet (or "Web") their favorite method of obtaining information and exchanging opinions. A mobile Internet era has arrived and concomitant reductions by major mobile communications corporations for $4 \mathrm{G}$ Internet access have made mobile communication a cost effective option for most people. The popularization of online videos and mobile Internet has led to prosperity for the video industry and continued evolution of communication modes.
In this context, the market for mobile short videos is undergoing rapid development in the video industry. Compared to traditional videos, mobile short videos have the advantages of instant dissemination, information concentration, direct connection with social platforms, and integration of production and sharing. Using the Meipai app of mobile short videos as a case study, this paper applies Lasswell's 5W model to mass communication using mobile short videos and suggests further development in mobile short video communications to further enhance its communication effectiveness.

Change in the Communication Environment with the Development of Mobile Internet

Current mobile Internet and mobile communications technology 


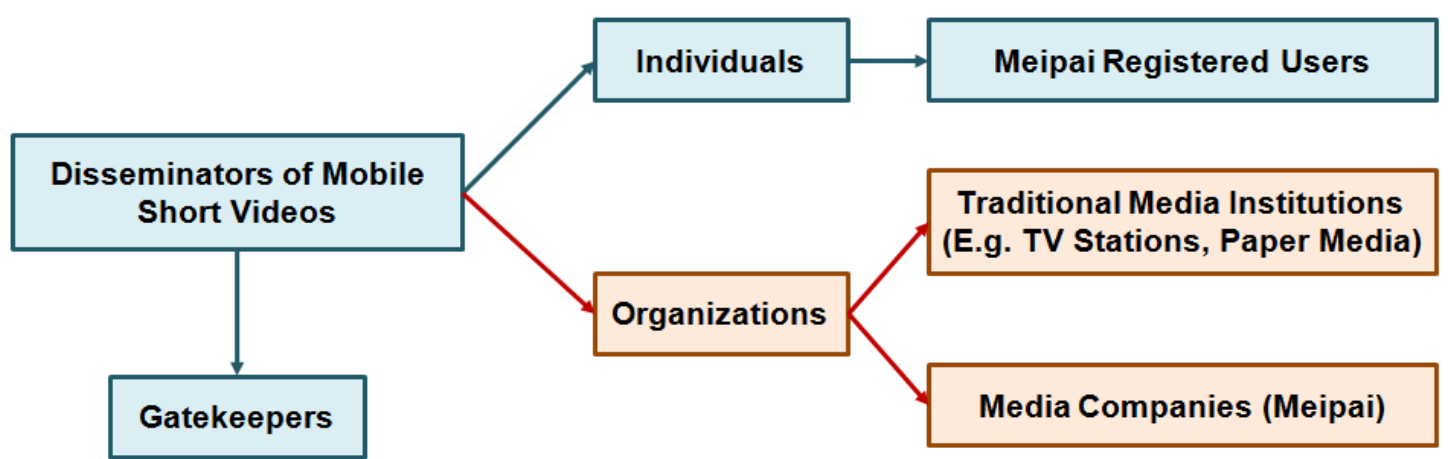

Figure 1. Communicators of Mobile Short Video in Meipai App.

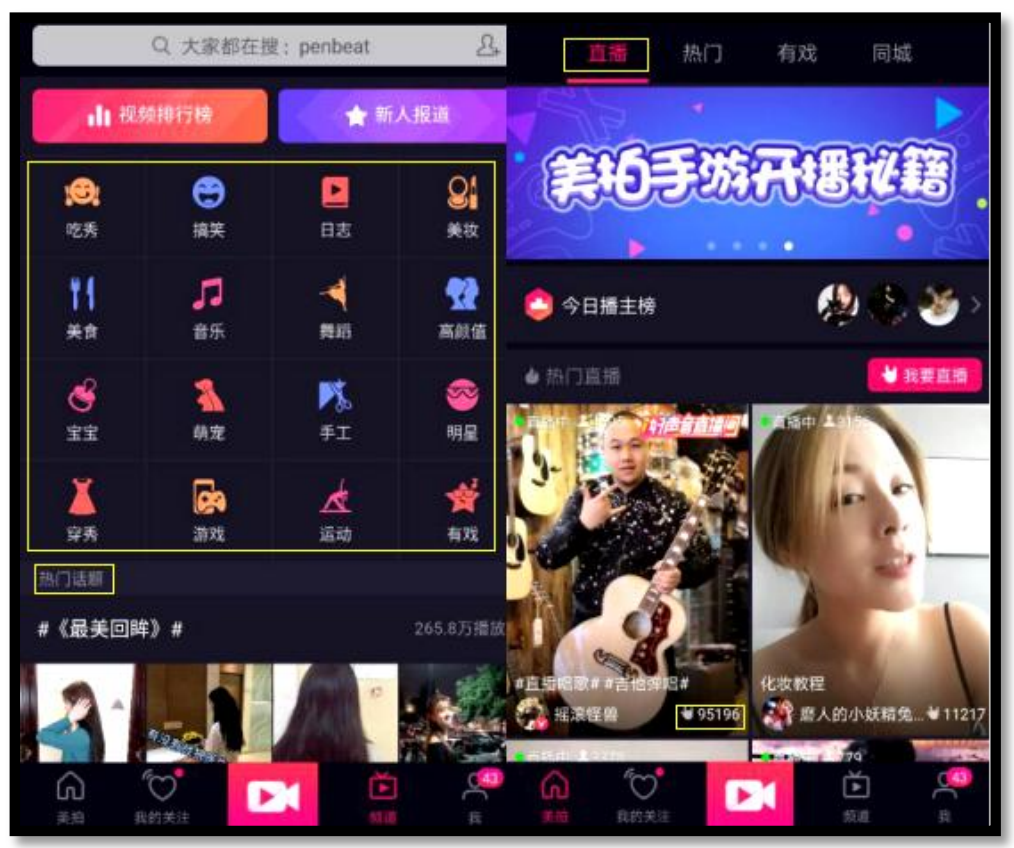

Figure 2. Channels and Live Broadcast Interface of Meipai.

allows the public to connect their mobile devices to the network anytime and anywhere to obtain the audio and video resources they need. In this virtual social network of Internet media, users can stay online and share high-quality content for entertainment in their spare time, disseminate newly generated information, including original content of their own creation and provide comments and feedback on others' information. In this network, an individual can act as a leader of opinions, by offering content they publish, comment on or forward, providing other people with the information needed to update microblogs, moments and the Chinese social networking app Qzone (1). Moreover, users can forward content found on their mobile phone to computers and televisions through the mobile network, so that when they $\log$ in to a computer or watch television with the same account, they see the same content as on their mobile phone. Inspired by these changes in the communication environment created through mobile Internet development, this paper further explores the future of such communications.

\section{Mobile Short Videos Definition of Mobile Short Videos}

The mobile short video is a new media trend triggered by the progress of new technology and popularization of the mobile Internet. At present, the definition of mobile short video varies. Baidu Encyclopedia (2) defines it as video content communication on new Internet media with duration of generally less than 5 minutes. In this paper, a mobile short video is defined as a short video, recorded and edited with a mobile smart phone, with a 


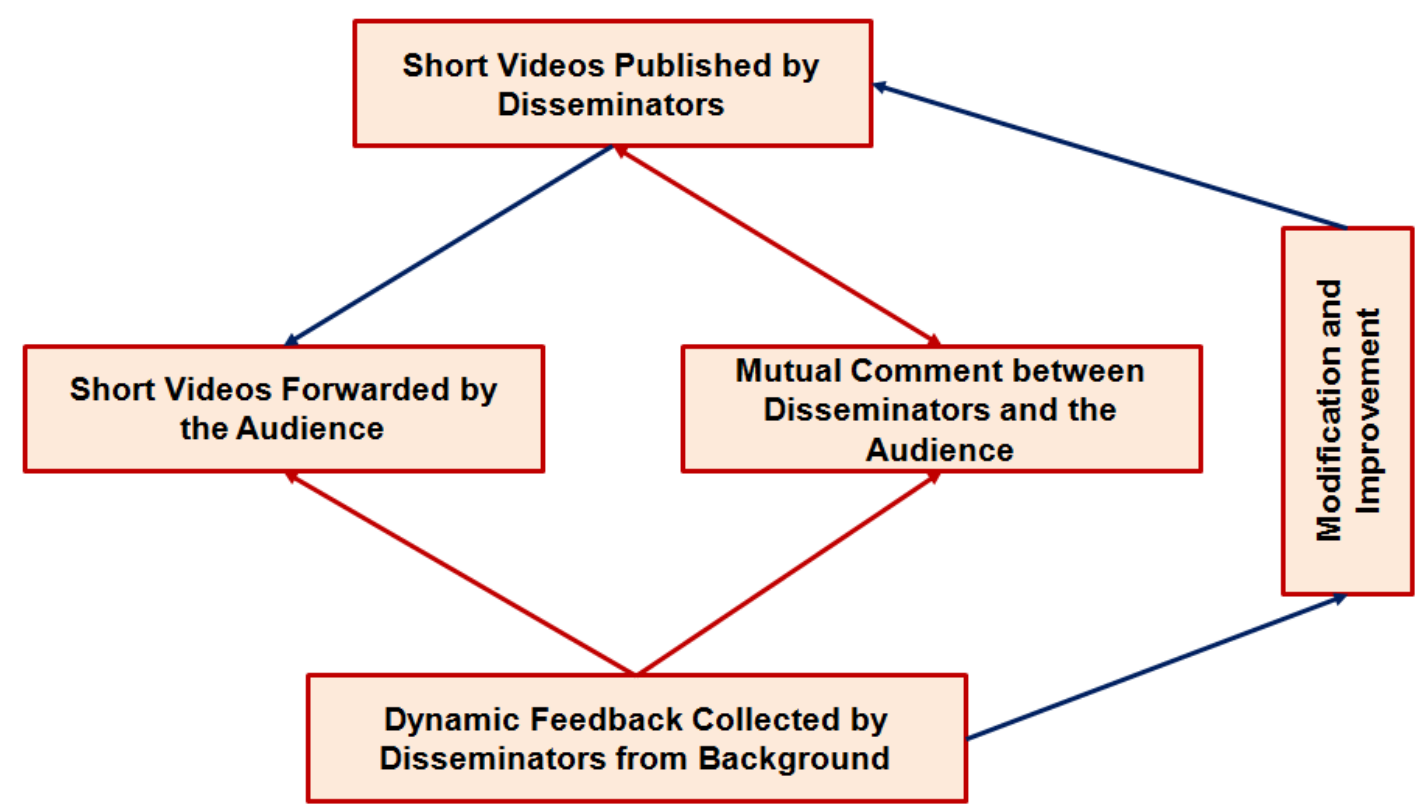

Figure 3. Feedback Procedure in Meipai Mobile Short Video Communication.

duration typically limited to within 10 minutes and shared on social media platforms through $4 \mathrm{G} / 5 \mathrm{G}$ or a Wi-Fi wireless network.

\section{The Developmental Trend of Mobile Short Videos}

Short videos began appearing in 2013. Almost at the same time, came the development of $4 \mathrm{G} / 5 \mathrm{G}$ and wireless networks and the popularization of smart phones. People began using mobile phones to record and edit videos and began to extensively watch videos on mobile phones. Statista, an international database company, notes that global mobile video traffic has risen steadily over the past six years and is now the main driving force for the growth of network data. Video traffic accounted for more than half of the total mobile data traffic in 2015 and continues to grow quickly (3). In China, mobile video has also developed rapidly. By 2018, many mobile short video apps had emerged and production had been serialized. These changes reflect the maturation of short video content in China

\section{Analysis of Mass Communication with Mobile Short Videos}

The transmission of mobile short videos is a communication activity in which short video transmission, acceptance and feedback take place among people, groups, organizations and society through the mobile $4 \mathrm{G}$ or wireless Wi-Fi network.

\section{Lasswell's 5W Communication Model}

In 1948, Harold Lasswell (4), an American political scientist, formally proposed five basic elements of communication for the first time, namely 'who,' 'say what,' 'in which channel,' 'to whom' and 'with what effect.' As the initials of the keywords in all the five elements begin with 'W,' this communication theory is referred to as the $5 \mathrm{~W}$ model.

\section{Case Study of the 5W Communication Model Applied to Mobile Short Videos on the Meipai App \\ W1: Who}

In mobile short video communication, the first element 'Who' is the disseminator of the video. Disseminators are responsible for collecting, sorting, selecting, processing, and transmitting information in the process of communication. They are called 'gatekeepers' and their performance is called 'gatekeeping' (5). The disseminators of mobile short videos might be individuals or organizations, including video lovers, traditional media institutions (such as TV stations, paper media, etc.) and film and television production companies. The communication is undertaken jointly by the disseminators and the audience.

As Figure 1 shows, disseminators may be registered users of the Meipai app. After registration and logging in, they can use the Meipai app to shoot, edit, upload, and share videos. In addition, disseminators may be traditional media personnel (TV studio, newspaper reporters, etc.). For example, when Shenzhou 11, a manned spacecraft, was launched in Jiuquan City, CCTV and China People's Daily reporters used the Meipai app on smart phones to shoot a short video of Shenzhou 11's ascent at the launch site, uploaded it immediately and shared it through the mobile Internet. As a result, people all over the country knew about the event instantly. Finally, professional media companies such as Meipai are also disseminators. Moreover, the Meipai team employs special reviewers to check and control the pro- 
duction of short video content. The reviewer inspects each uploaded video, within one-hour of posting time. Any video with uncivilized content is deleted. Those approved will be uploaded to the Web in time. Therefore, on mobile Internet, the disseminator of mobile short video can be anyone who can make videos Nonetheless, it is essential that the disseminator performs his or her role as a gatekeeper by collecting only appropriate sources and producing high quality, valuable videos to achieve good communication results and to obtain good feedback.

\section{W2: What}

The second element in the $5 \mathrm{~W}$ model is 'say what' - the content or information. In the case of the Meipai app, the communication content is the video images recorded with the Meipai app on a smart phone.

\section{Characteristics of Mobile Short Video Content: Short, Original and Life-Like}

People are increasingly dependent on mobile phones, keeping them on their person at all times. Thus, mobile phones move with people and this is where their mobility comes from. However, despite their indispensable and mobile nature, videos communicated through mobile phones tend to be short, for two reasons. One is that the screens of mobile phones are relatively small compared with that of other electronic devices; spending a long time watching mobile video becomes difficult on the eyes. The other, more important, reason is the limitation of the mobile's phone standby time. Video works shot with the Meipai app typically last 10 seconds to 5 minutes. Meipai's slogan is, "A blockbuster can be shot in 10 seconds - Meipai is a super fun short video community" (6). Producers of mobile short videos pursue originality. The originality of Meipai short videos comes from its user generated content (UGC) mode, which stimulates the enthusiasm of Internet users to participate in video creation. In addition, the desire to produce original videos motivates users to search for sources from real life, enhancing authenticity of Meipai mobile short videos.

\section{Content Variety: Diverse Categories}

At present, mobile short video content has diverse and numerous categories. In the case of Meipai, mobile short videos are classified into many channels. By September 2017, Meipai had 16 channels and a hot topic zone, as shown in Figure 2. In addition, with the increased coverage of the mobile Internet, the live broadcast application in the Meipai app has become a new favorite among users. The number of people simultaneously watching a live broadcast can reach as high as 95196. Furthermore, as soon as Meipai users upload videos onto the mobile Internet, they can find their own video works on the server and share them with friends, who can see them immediately. People can also see videos shared and uploaded by others in the Meipai app. Different users observe life from different angles and produce different content, thereby creating a wide variety of short videos. Mobile short video application platforms should collect information on the categories of videos watched by users and analyze the data, so that they can produce and push short videos with content catering to public tastes.

\section{W3: Which Channel}

The third element in mobile short video communication is the avenues used for information transmission. Although cell phones are the main vehicle for mobile short video communication, other technology is also required, namely the $4 \mathrm{G} / 5 \mathrm{G}$ network or Wi-Fi wireless networks, and these play a large role in increasing popularity for Meipai worldwide.

\section{Mobile 4G/5G Network}

In 2014, the Internet witnessed the arrival of $4 \mathrm{G}$, providing improved connections to the internet from mobile devices and making communication among friends more convenient. According to survey data from the China Internet Information Center (CNNIC), by December 2016, China's mobile Internet users had reached 695 million. Major domestic Internet companies that have video websites are optimistic about mobile Internet business and have been launching many products to seize on this business opportunity (7). At present, China is starting trials of a $5 \mathrm{G}$ network and $4 \mathrm{G}$ networks are increasingly popular. More audiences at different ages use mobile networks to watch Meipai videos and other short videos. Thus, the 4G/5G network provides an avenue for faster access to mobile short videos.

\section{Wi-Fi Wireless Network}

With the rapid development of the Internet, wireless internet access has become a common part of people's daily life. Wi-Fi, an abbreviation of 'wireless fidelity,' is typically referred to as the wireless network. Wireless networks have many advantages, including ultra-fast audio and video signal transmission and wide coverage, creating another effective avenue for mobile short video communication. Users in areas covered by a wireless network can effortlessly access the network to upload, watch, share and forward mobile short videos anytime through applications like Meipai; using Wi-Fi technology can greatly reduce time-lag between recording video and uploading video.

These two avenues for mobile short video communication play an important role in promoting the development of short videos. However, they are insufficient and new avenues for mobile short video communication should be explored.

\section{W4: To Whom}

The audience, the receiver of information, is the fourth element in communication. The audience of mobile short videos is the cell phone users who watch mobile short videos and an analysis of this demographic can improve the results of mobile short video communication. The network audience's choice of mobile short videos depends on their own preferences, interests and emotional expressions of the videos. The major producers of Meipai short videos can be classified into three types. (i) Meipai users who shoot mobile short videos as a hobby. They are trying to make films out of their daily life and hope the audience will appreciate them. (ii) Recorders of major events such as conferences, important games and concerts with the Meipai app for special usage. (iii) Meipai users with specific skills, such as cosmeticians, craftsmen, and chefs, who record tutorials to help audiences learn these skills with the Meipai app. For maximally effective mobile short video communication, producers must consider the needs of the audience when shooting, editing and 
classifying short videos.

\section{W5: With what Effect}

Feedback from communication, the fifth element in the $5 \mathrm{~W}$ model, refers to comments made by audiences after receiving information from the disseminators. In mobile short video communication, the disseminator can modify and improve the content of a mobile short video based on feedback from the audience. Communication thus becomes an interactive practice between the disseminator and the audience. With the mobile Internet and a platform with a feedback system, the viewers of mobile short videos are provided with an easy way to transmit comments to the disseminator, improving the efficiency and effectiveness of communication.

Each Meipai registered user is allocated an account, through which they can search for and add other accounts for mutual following. As Figure 3 shows, if a Meipai user likes a mobile short video, they can post comments on the short video and, more importantly, introduce and recommend the short video to people around them, and even forward it to other social media platforms for promotion. By collecting dynamic information from users, the staff of Meipai or the disseminator can see people's responses to this short video and judge whether to modify the video or whether to publish similar videos again. The analysis on Meipai's reaction of the audience indicates that if an app keeps following feedback from the audience, the flow of mobile short video communication will remain smooth, which will attract more fans of mobile short videos.

\section{Potential Development in Mobile Short Video Communication}

With the benefit of the mobile Internet, mobile short video communication has built a large audience, met the various needs of users, integrated communication avenues and developed flexible and diversified communication modes. Despite these advantages, shortcomings exist. Prospects of development in mobile short video communication are predicted as follows.

\section{Role Interchange between Disseminators and Audiences}

According to the above analysis, disseminators effectively control the direction of information flow and the response of audiences. Viewers are the information receivers in the communication and have the right to filter and reject information. However, the separation of the two roles should be removed. Users of mobile short video applications can be both producers and viewers. Therefore, the roles of disseminator and audience should be interchangeable.

\section{Integration of Mobile Short Video with Educa- tional Resources}

By 2016, domestic mobile short video applications had been introduced into the daily life of the Chinese public. In Meipai short video content, the channels are mainly related to everyday life and entertainment. In future, the scope of mobile short video content should be expanded. For example, in the context of educational system reform in China, a meaningful experiment would be to apply mobile short video communication to China's education. As an example, teachers can use smart phones to shoot their introduction of the new lesson before class and then edit the video with the tools provided by the Meipai app before uploading it to the Internet. In this way, students can learn by themselves through short videos before class and bring questions to class. Using class to answer questions, discuss the material and solve problems, students will acquire a deep understanding and memory of the new knowledge from the current lesson. By this teaching method, mobile short video communication could help improve student learning outcomes.

\section{Developing Multi-Avenue Communication}

At present, full coverage of mobile Internet in rural and mountainous areas continues to face problems such as high transformation costs and a shortage of investment. To popularize mobile short videos nationwide, it is necessary to develop multiple avenues of communication, speed up the construction of the Internet and ensure the signal reception quality on mobile Internet. Paper leaflets and mobile phone advertising messages might be feasible ways to introduce the concept of mobile short videos and the methods of making original short videos so that more people will participate in mobile short video production and transmission.

Rapid development in the mobile Internet leads to increasing convenience in interpersonal contact and communication. This new communication environment will motivate Netizens to devote more time and energy to producing original mobile short videos. Those loved by the mass audience will circulate widely. To promote the fast and healthy growth of mobile short video communication, it is strongly recommended that more people should be encouraged to participate in this practice both as disseminators and audience members, that multiple communication avenues are developed by experts in mobile technology, and that mobile short videos be integrated into educational activities.

\section{References}

1. Miao HY. Communication Research on the Rise and Development of Chinese Microfilm under the Background of Mobile Internet. Beijing: Beijing University of Posts and Telecommunications Press. 2013.
2. Baidu Encyclopedia. Retrieved from: https://baike.baidu.com/. 2017-12-13.

3. Wang $\mathrm{XH}$, Ren YT. New features and problems of short video production in china. News Front 2016; 
2016(17):4. DOI:

https://doi.org/cnki:sun:xwzx.0.2016-17-034

4. Lasswell $\mathrm{H}$. The Structure and Function of Commu nication in Society. Beijing: Communication University of China Press. 2013.

5. Liang J. Analysis on Lasswell's 5W Model -- in the case of TV Column "Half the Sky". Knowledge Econ
2013; 2013(3):1.

6. Live Channel-Meipai. Retrieved from: http://www.meipai.com/live. 2017-12-13.

7. Zhong JJ. The art of mobile digital images. Qufu Norm Univ 2010. DOI:

https://doi.org/10.7666/d.Y9014568 